\title{
Heat Transfer in the Boiling Regime of Impinging Liquid Jets
}

\author{
Steffen Waldeck ${ }^{1}$, Udo Fritsching ${ }^{2}$ \\ ${ }^{1,2}$ University of Bremen, Particles and Process Engineering \\ Badgasteiner Str. 3, Bremen, Germany \\ s.waldeck@iwt.uni-bremen.de \\ ${ }^{2}$ Institute of Materials Science \\ Badgasteiner Str. 3, Bremen, Germany \\ ufri@iwt.uni-bremen.de
}

\begin{abstract}
Water jets may be utilized as rapid cooling devices in heat treatment and casting processes of metallic specimen. Here, at surface temperatures above the boiling and the Leidenfrost temperature, the liquid partially evaporates in different stages. Due to these boiling regimes the local heat flux strongly varies. To investigate the heat transfer process during flow boiling of impinging jets and the resulting cooling mechanisms, experimental investigations with high speed camera as well as infrared camera were performed. A numerical model has been developed that enables to simulate the coupled multiphase and heat transfer processes in the entire quenching process with all boiling phenomena and cooling stages. The model is based on the Eulerian approach for multiphase flow. To calculate the heat and mass transfer during partial liquid evaporation (e.g. bubble and film boiling) the conservations equation were supplemented with sink and source terms for evaporation and condensation. The results of boiling regime behaviour and the heat transfer coefficient (HTC) especially at the Leidenfrost point are discussed. The HTC at Leidenfrost decreases with increasing distance to the impingement point. A correlation for the local Nusselt number $\mathrm{Nu}$ is derived.
\end{abstract}

Keywords: Quenching, Impinging Water Jet, Leidenfrost effect, Boiling Phenomena

\section{Introduction}

In heat treatment or shaping processes of metallic semi-finished products and structural parts, water jets or sprays are often used for an intensive and controlled quenching. Typical surface temperatures range from above $1000{ }^{\circ} \mathrm{C}$ for steel alloys or up to $600{ }^{\circ} \mathrm{C}$ for aluminium alloys in order to obtain the desired cooling progress and the required material and component properties. An important requirement for the cooling process is the controlled setting of a spatially and temporally defined course of the heat transfer or heat flux in order to avoid temperature inhomogeneities in the component. Due to the surface temperatures to be quenched, flow boiling takes place which causes uneven heat transfer rates for the different boiling phases. Here film boiling above the Leidenfrost temperature, transition and nucleate boiling are occurring simultaneously or in a subsequent manner [1]. The intense evaporation may lead to lift off and splashing of the water film from the hot surface.

\section{Experimental Research}

In experimental investigations, the spreading of the wetting front is tracked with recordings of a high-speed camera. The temperature profiles are measured with an infrared camera [2]. Here, thin stainless steel plates $(200 \times 200 \mathrm{~mm})$ with thickness of $2 \mathrm{~mm}$ were heated in an oven to initial temperatures of 550 to $850{ }^{\circ} \mathrm{C}$. The plates are quenched with water jets from nozzles (outlet diameters of 0.8 to $1.3 \mathrm{~mm}$ ) impinging onto the centre of the plate. Jet velocities were varied from 10 to $30 \mathrm{~m} / \mathrm{s}$ thus leading to Reynolds numbers in the range from about 10.000 to 40.000 . The distance between the nozzle and the plate is held constant at $40 \mathrm{~mm}$. The water temperature is set to $22{ }^{\circ} \mathrm{C}$. To determine the local temperature distribution, the backside of the plate is recorded by an infrared camera. From the temperature field evolution the heat transfer coefficient on the frontside (impinging side) of the plate is deduced.

Figure 1 shows a snapshot of the impinging jet on the hot surface. The water jet impinges the plate from above. From the centre the rewetting front starts moving radially outwards. In the area close to the impingement point, the surface at the time shown is already wetted. The bright area indicates the nucleate and transition boiling regimes in these area. Due to the 
perpendicular momentum caused by the liquid evaporation onset, the water film lifts off from the surface at the Leidenfrost point and forms droplets. These droplets slide outwards without wetting and practically without cooling the surface. From these visual observations the wetting front velocity was determined. The wetting front velocity as well as the temperature profiles have been used as essential parameters for the validation of the numerical model.

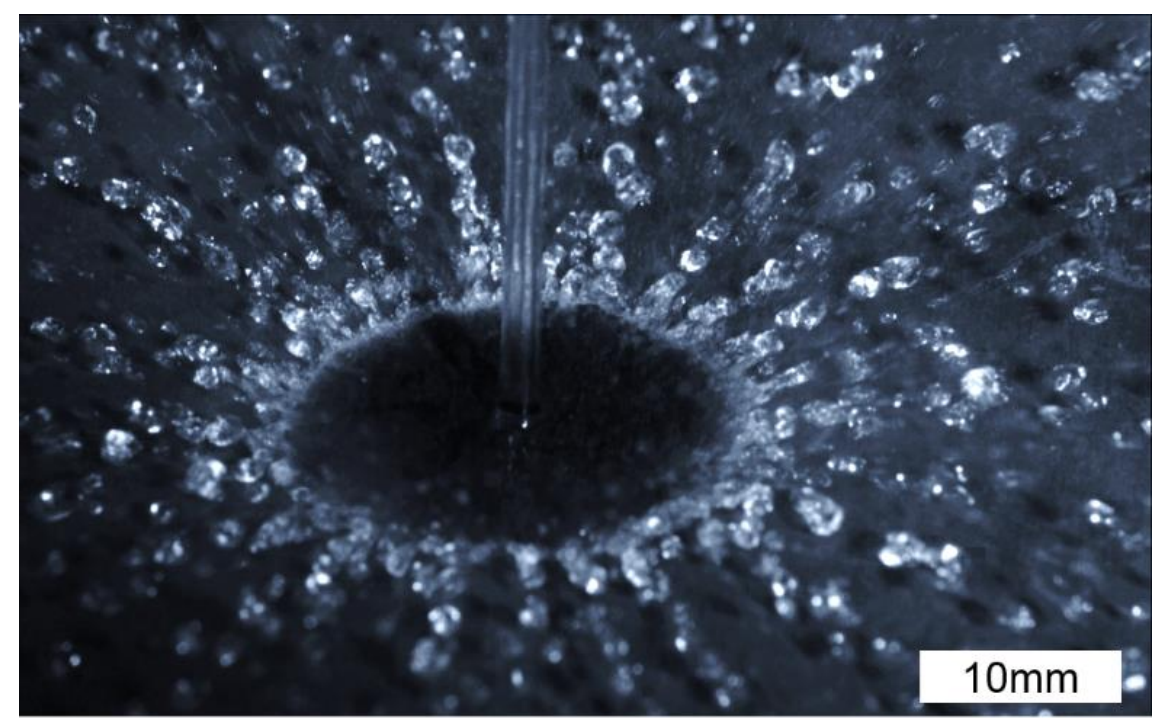

Fig. 1: High speed camera image of an impinging water jet on a hot surface.

\section{Numerical Simulations}

The numerical model of evaporating liquid flow is based on the Eulerian multi-phase model. The classical approach is extended by sink and source terms for the ability to calculate the boiling phenomena [3, 4]. In the Euler model the phase boundary between liquid and vapour is not calculated directly, it is somewhat smeared over some cells of the mesh (diffuse interface). A volumetric phase coefficient $(\alpha)$ ranging from 0 to 1 is calculated for each cell for each time step. From these coefficients in the cells located directly above the cooled area, the local boiling phase can be derived. Figure 2 shows schematically the local boiling phase related to $\alpha$.

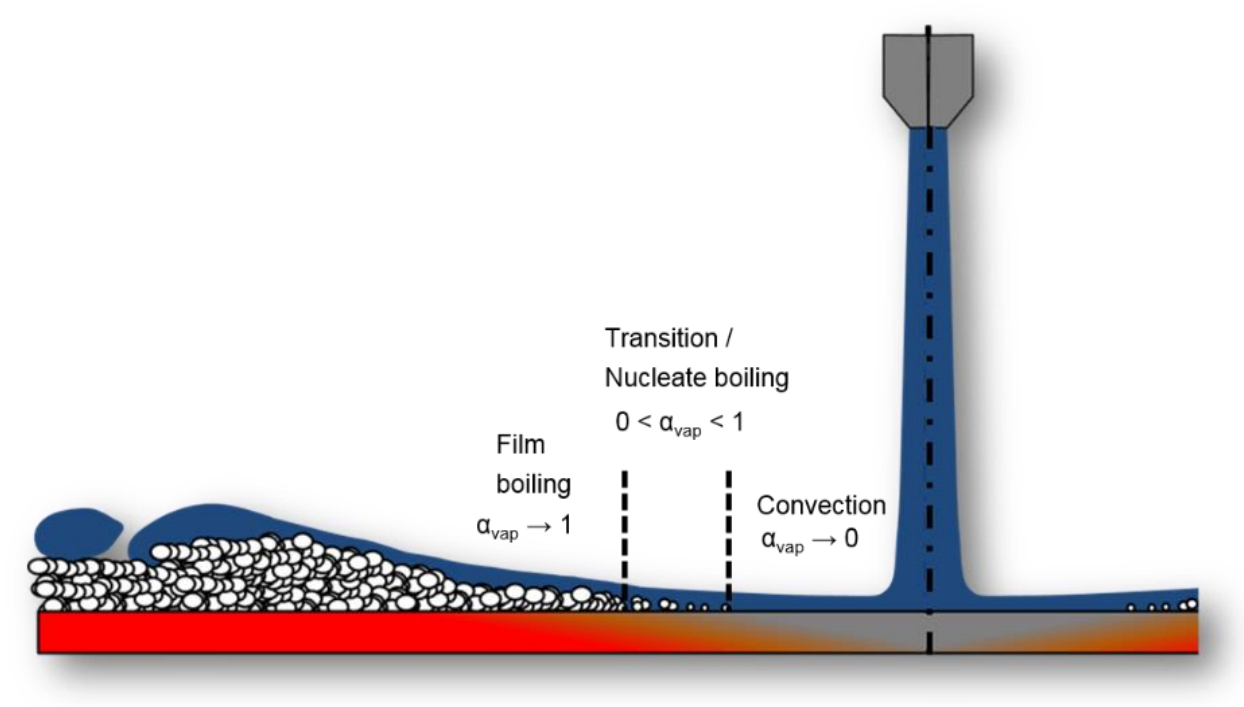

Fig. 2: Schematic visualization of the volumetric phase coefficient and boiling regimes. 
With this model the whole quenching process can be simulated continuously. In addition to the variables of the temperature distribution and the wetting behaviour, which are used for the validation with the experiments, further variables of the thermodynamics and hydrodynamics can be derived. The heat flux or the heat transfer coefficient can be considered without the errors of an inverse calculation as well as the water film thickness, speed profiles and local pressure distribution can be analysed.

\section{Results}

Figure 3 shows the heat transfer coefficients at the Leidenfrost point as a function of the dimensionless distance to the jet axis. The results are from simulations with a $1.3 \mathrm{~mm}$ nozzle diameter. Varied parameters were the outlet velocity and the initial plate temperature.

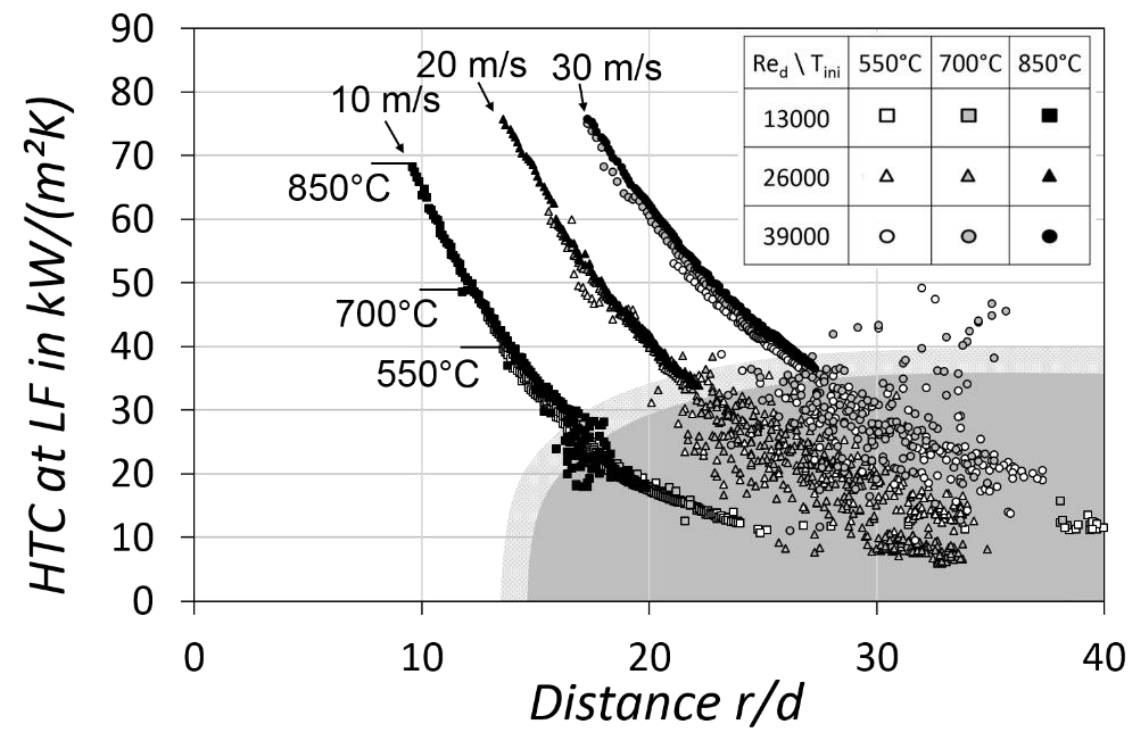

Fig. 3: HTC over dimensionless distance to the jet axis for nozzle diameter of $1.3 \mathrm{~mm}$ and varied process parameters.

It can be seen from the curves that the HTC decreases with increasing dimensionless distance. There is a small temperature dependency on the slope of the curves, but the starting point is shifted downwards for lower temperatures. With an increased exit speed, the curves shift to the right. The illustration shows that the process takes place in three different phases. In the area near the impinging point the HTC drops almost linearly. This area appears to be determined by the hydrodynamics of the water jet. Subsequently, a transitional area is recognizable (slightly grey background). The third area (grey background) in the far field of the water jet shows way more scattered values for the HTC. In this area, the process is supposed like water wall film. The special nature of the water jet has no further influence. The scattering is due to a step-like progress of the wetting front and the associated numerical inaccuracies.

In order to derive a correlation to calculate the local heat transfer rate from these results, the essential influencing variables have to be determined. From figure 3, the outlet velocity, the distance to the jet axis, the nozzle diameter, the fluid properties (viscosity, density, thermal conductivity and surface tension) are derived as crucial parameters for the HTC. These process variables are expressed in the dimensionless quantities $\mathrm{r} / \mathrm{d}$ as well as the Nu-, Re-, and Oh-numbers. By means of a suitable selection of the axes and with the aid of these parameters the results of the different simulations can be mapped onto only one curve (Figure 4). In addition to the results from Figure 3, more values were varied and simulated.

The course of this master curve can be described by an exponential function. This results in an empirical correlation for the considered parameters for the prediction function of the nozzle diameter-related Nu number or rather the calculation of the HTC:

$$
N u=4 \cdot 10^{-9} \cdot R e^{0,8} \cdot(r / d)^{-1,4} \cdot O h^{-3,5}
$$


It is noted that this correlation is only valid for the examined process variables. Other parameters such as the material influences, different sizes of nozzle diameters and other fluids are part of current research activities.

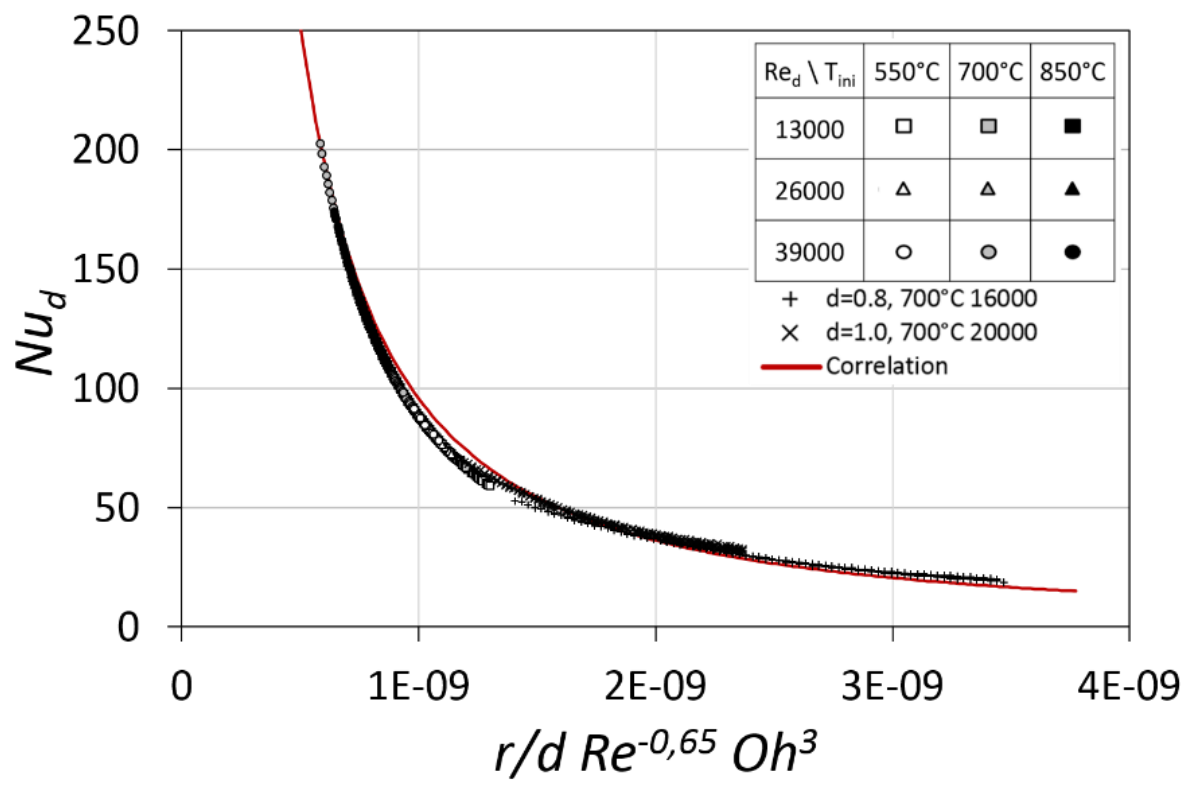

Fig. 4: Master curve for Nu number correlation for varied process parameters.

\section{Conclusion}

The heat transfer at hot surfaces at temperatures above the Leidenfrost temperature during intensive quenching with water jets has been investigated experimentally and numerically. For this purpose, experiments were made on thin steel plates with an HS camera for tracking the wetting front and a temperature distribution was measured with an IR camera. The results were used to validate an enhanced numerical simulation model. This model allows to simulate the whole quenching process within all boiling phenomena. The model enables the investigation of the local heat transfer mechanisms directly. It is shown that the course of the HTC decreases over the distance to the impinging point and how individual parameters such as nozzle outlet velocity and initial plate temperature influence the heat transfer in the different boiling regimes. With the help of dimensionless numbers, results from simulations with different parameters could be projected onto one master curve. From this curve, a correlation was determined for the Nu number respectively for the HTC.

\section{Acknowledgements}

This research has been conducted within a project funded by AiF through resources of the BMWi.

\section{References}

[1] S. Nukiyama, "The maximum and minimum values of the heat q transmitted from metal to boiling water under atmospheric pressure," J. Heat Mass Transfer., vol. 9, pp. 1419-1433, 1936.

[2] A.K. Nallathambi, E. Specht, "Estimation of heat flux in array of jets quenching using experimental and inverse finite element method," J. Materials Processing Technology, vol. 209, pp. 5325-5332, 2009.

[3] S. Waldeck, U. Fritsching, "Multiphase Investigation of Intensive Quenching with Waterjets," in Proceedings of the ICMF International Conference, Firenze, IT, 2016, vol. 9.

[4] P. Stark, "Prozesssimulation der Bauteil-Abschreckung in flüssigen Medien: Zur Analyse des mehrphasigen Strömungs- und Wärmetransports in Siedeprozessen" Ph.D. dissertation, IWT, Univ. Bremen. 